\title{
Analysis of the binding mode of a novel HIF1a inhibitor through molecular modelling
}

\author{
Yi Zhao ${ }^{1 *}$, Ying $\mathrm{Li}^{1 *}$, Ju Hong Wang ${ }^{1}$, Jia Zhang ${ }^{1}$ and Xu Zhang ${ }^{2}$ \\ ${ }^{1}$ Department of Oncology, The First Affiliated Hospital of Dalian Medical University, Dalian, Liaoning, Zhongshan \\ Road 222, Dalian, Liaoning 116011, P.R. China \\ ${ }^{2}$ Department of Thoracic Surgery, The Second Affiliated Hospital of Dalian Medical University, Zhongshan Road 467, Dalian, \\ Liaoning 116023, P.R. China
}

\begin{abstract}
Hypoxia inducible factor-1 (HIF1) is an important transcription factor related with tumor metastasis. As a subunit of HIF1, HIF1a plays an important role in regulation of the hypoxic response. HIF1 $\alpha$ inhibitor could be a promising treatment for certain cancers. In the present study, we try to model the binding mode of the recent reported new series of HIF1a inhibitors with the purpose of further improving the performance of these inhibitors. Molecular docking was first employed to predict the binding modes of the protein-ligand complexes, followed by molecular dynamics simulations and MMGBSA free energy calculations. According to the predicted binding modes, these molecules form two important hydrogen bonds with HIF1a. Moreover, if the molecules could form a stable $\pi-\pi$ interaction with HIF $1 \alpha$, the potency of the inhibitors can be greatly improved. Hydrophobic interactions between the molecules and HIF1 $\alpha$ are also a key factor, especially the interactions between the hydrophobic groups (benzimidazole) of the molecules and the hydrophobic residues inside the binding cave. Molecular modeling could be a useful method in the future drug design. In this study, our calculation can help to design and develop high potent HIF1a inhibitors in future.
\end{abstract}

Key words: Molecular docking - Molecular dynamics - Inhibitor - Cancer - HIF1a

\section{Introduction}

Caner is currently the second leading cause of death in the world, with around 8.8 million deaths in 2015, or 1 in 6 deaths is due to cancer (Forouzanfar et al. 2015).

The identification of novel targets for cancer medication is now a high priority. Many studies have been carried out with emphasis on effective signal pathway. Among these studies, hypoxic cell signaling has attracted many attentions, as cancer cells have different behaviors with normal cells, such as the different oxygen levels. These abnormal oxygen levels would trigger the activation of survival pathways which would not be triggered in normal cells. So this pathway

Correspondence to: Xu Zhang, Department of Thoracic Surgery, The Second Affiliated Hospital of Dalian Medical University, Zhongshan Road 467, Dalian, Liaoning 116023, P.R. China

E-mail: zhangxuhealthy@mail.com

* Both authors contributed equally to this work could be a promising direction in developing the anti-cancer medication (Brown and Wilson 2004).

Hypoxia inducible factor-1 (HIF1) discovered in the early 1990s is an important transcription factor related with tumor metastasis (Semenza 2000, 2001, 2003; Onnis et al. 2009). HIF1 regulates the transcription of hundreds of genes in a cell type-specific manner (Semenza 2000, 2001,2003 ). The mechanism studies provided a molecular target associated with intratumour hypoxia that could be used for the development of novel cancer therapeutics (Majmundar et al. 2010).

As shown in Figure 1, HIF1 is a heterodimer transcription factor belonging to the basic-helix-loop-helix protein superfamily, composed of HIF1 $\alpha$ and HIF1 $\beta$ subunits (Semenza 2007). HIF1a serves as a regulator of the hypoxic response, including in cancer cells, through transcriptional activation of several target genes (Wang et al. 1995). Intratumoral hypoxia and genetic alterations can lead to HIF1a overexpression, which has been associated with tumor angiogenesis, 


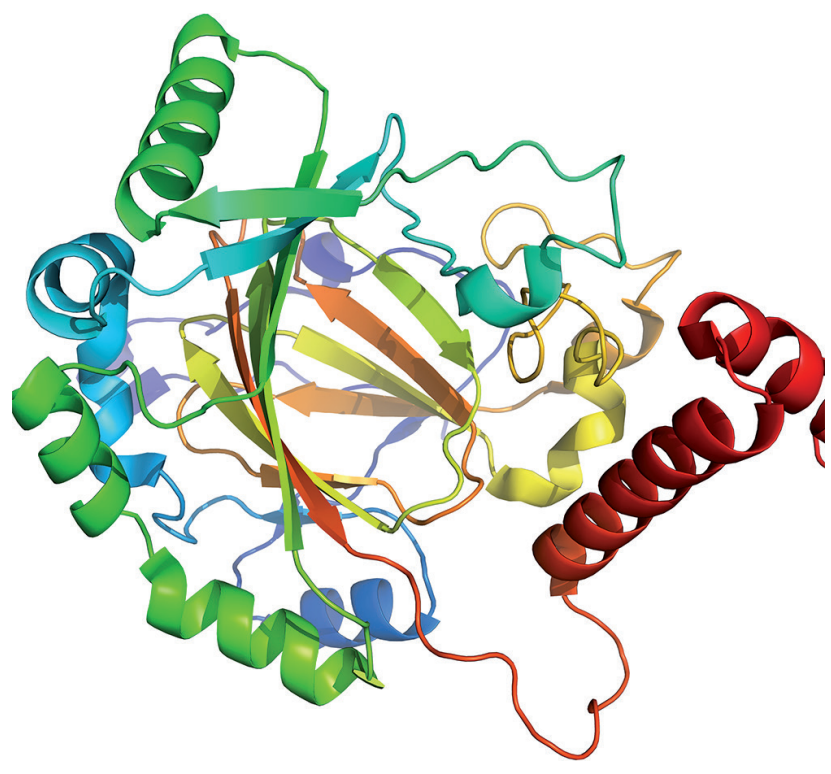

Figure 1. Overview of HIF1a structure in color ribbon cartoon model. (See online version for color Figure 1).

tumor initiation, progression, invasion, metastasis, and drug resistance (Semenza 2003). So HIF1a has becoming an important target and is attracting more and more attention (Sullivan and Graham 2007; Soeda et al. 2009).

HIF1 $\alpha$ is a protein composed of 352 amino acid residues (Semenza 2007). Considering its important role in many cancers, developing a high efficient HIF1a inhibitors is becomes a priority (Yang et al. 2016). After years of effort, many kinds of inhibitors were designed and tested. However, due to the intrinsic challenges associated with the discovery and development of pharmacological inhibi- tors of transcription factors, such as the lack of specificity, currently there is no Food and Drug Administration (FDA) approved drug (Giaccia et al. 2003; Onnis et al. 2009; Chen et al. 2014). Recently, one of these studies discovered a new series of HIF $1 \alpha$ inhibitors derived from the YC-1 inhibitor (Hsu et al. 2003; Yeo et al. 2003; Wang et al. 2005; Masoud et al. 2015). According to this study, these novel phenelzine analogues have been shown to be potent HIF1a inhibitors.

In the present study, we are focusing on how to improve the performance of these novel HIFla inhibitors by using computer aided drug design method. To achieve this, we first need to understand how these inhibitors bind with HIF1 $\alpha$ by using molecular docking method. The predicted binding mode of these compounds were followed by molecular dynamics simulation and MMGBSA binding free energy calculation which can give us a more accurate description of the mechanism of the binding. With all these insight information, it would be highly possible to conduct the rational drug design which would lead to the development of a promising anti-cancer medication based on HIF1a inhibitor.

\section{Materials and Methods}

\section{Protein preparation and ligand preparation}

The starting HIFla 3D structure was taken from the X-ray crystal structure deposited in Protein Data Bank at $2.5 \AA$ resolution (PDB ID: 4Z2W) (Taabazuing et al. 2016).

The 3D structures of the compounds listed in Figure 2 were prepared and minimized by Avogadro program with MMFF94s force field (Hanwell et al. 2012).<smiles>COc1ccc(CC(=O)NC2Nc3ccccc3N2Cc2ccccc2)cc1CN1C(NC(=O)Cc2ccc(F)cc2)=Nc2ccccc2N1Cc1ccc(Cl)cc1</smiles>

Figure 2. The structures of HIF1a inhibitors. 


\section{Molecular docking}

Molecular docking is a useful method in predicting: (1) the binding affinity between the ligand and the receptor which can give us a basic idea of the potency of the inhibitor; (2) the binding pose of the ligand which can give us a big picture how the ligand interacts with the receptor. In this study, molecular docking studies were performed through Autodock Vina (Trott and Olson 2010). The 3D structures of HIF1a (with waters and the other molecules in the crystal structure removed) and the ligands were processed by ADT program (Morris et al. 2009). The docking of the compounds was followed by the standard protocol of Autodock Vina. The docking box size is set to $30^{\star} 30^{\star} 30(\AA)$. For each docking, top 10 conformations (ranked by the binding free energy) were saved for later analysis.

\section{Molecular dynamics and MMGBSA}

Molecular dynamic simulations were simulated by using Amber 9 program package, ff03 force field was used to simulate protein and general AMBER force field (GAFF) was used to simulate small molecules (Case et al. 2006). The missing force field parameters of the ligand and the partial charges were calculated by Antechamber of Amber 9. The initial structures of the protein-ligand complexes (from molecular docking results provided by Autodock Vina) were processed using tleap of Amber 9 to generate the topology and coordinated files. Particle Mesh Ewald (PME) method was used to calculate the long-range electrostatic interactions (Essmann et al. 1995). To improve the simulation efficiency, SHAKE was used to constrain the lengths of all covalent bonds involving hydrogen atoms with a time step of $2.0 \mathrm{fs}$ (Ryckaert et al. 1977).

Now we need to add water to the simulation systems and heat the simulation systems to the body temperature. The receptor-ligand complexes were solved in an orthorhombic box using TIP3P water model with a minimum solute-wall distance of $10 \AA$ (William et al. 1983). The whole system was energy-minimized and gradually heated in the NPT ensemble from $10 \mathrm{~K}$ to $300 \mathrm{~K}$ over 60 ps. Then, Molecular dynamics (MD) simulation was performed for 5 ns under the normally adopted temperature $(300 \mathrm{~K})$. The atomic coordinates were saved every 1 ps for subsequent sampling and analysis.

The binding free energy gives us a more accurate description from energy point of view. The binding free energy calculations were performed by using the MM/GBSA module in Amber 9 (Case et al. 2006). The binding free energy includes three terms: van der Waals contribution $\left(\Delta \mathrm{E}_{\mathrm{vdW}}\right)$, electrostatic contribution $\left(\Delta \mathrm{E}_{\mathrm{ele}}\right)$, and solvation contribution $\left(\Delta \mathrm{G}_{\mathrm{GB}}+\Delta \mathrm{G}_{\mathrm{SA}}\right)$, where $\Delta$ Evdw and $\Delta$ Eele are nonbonded van der Waal interaction and electrostatic interaction between the inhibitor and protein, which can be computed using the sander program in Amber 9 (Case et al. 2006).

$$
\Delta \mathrm{G}_{\text {inhibitor-protein }}=\Delta \mathrm{E}_{\mathrm{vdW}}+\Delta \mathrm{E}_{\mathrm{ele}}+\Delta \mathrm{G}_{\mathrm{GB}}+\Delta \mathrm{G}_{\mathrm{SA}}
$$

The polar contribution $\left(\Delta \mathrm{G}_{\mathrm{GB}}\right)$ of desolvation was computed using the generalized Born (GB) model, and the nonpolar contribution of desolvation $\left(\Delta \mathrm{G}_{\mathrm{SA}}\right)$ was computed using the surface area. The charges used in GB calculations were taken from the Amber parameter set (Case et al. 2006). All energy components were calculated using 50 snapshots from 3 ns to 5 ns.

So in short summary, the protocol would be: (1) using molecular docking method through Autodock Vina to fast predict the possible binding mode of these HIF1a inhibitors with HIF1a and ranking the result using the docking energy, (2) followed by MD simulations through Amber 9 to generate the trajectories for later sampling, (3) MMGBSA (included in Amber 9) was used to calculate the binding free energy to further explain these binding modes.

\section{Results}

\section{Binding energy analysis}

To quickly evaluate these compounds, molecular docking studies were first performed to calculate the binding energy of the compounds with HIFla. The results are listed in Table 1. According to the experimental results, these seven compounds (named from $\mathrm{H} 1$ to $\mathrm{H} 7$ for easy noting, see Figure 2 and Table 1 for detail) have the docking energy ranging from -9.6 to $-10.2 \mathrm{kcal} / \mathrm{mol}$. The correlation between the Auodock Vina Energy and experimental value is moderate with $\mathrm{R}^{2}$ equals 0.60 .

\section{Binding mode analysis}

To further investigate the interaction between these analogous and HIF1a, binding mode analysis were performed.

Table 1. The binding energies of phenelzine analogues

\begin{tabular}{ccc}
\hline Compound name & Vina Energy $(\mathrm{kcal} / \mathrm{mol})$ & $\mathrm{IC}_{50}(\mu \mathrm{M})$ \\
\hline H1 & -9.9 & 8.12 \\
H2 & -10.1 & 1.58 \\
H3 & -9.7 & 2.77 \\
H4 & -9.8 & 3.58 \\
H5 & -9.6 & 1.78 \\
H6 & -10.2 & 1.53 \\
H7 & -9.5 & 12.45 \\
\hline
\end{tabular}

$\mathrm{IC}_{50}$, half maximal inhibitory concentration. 


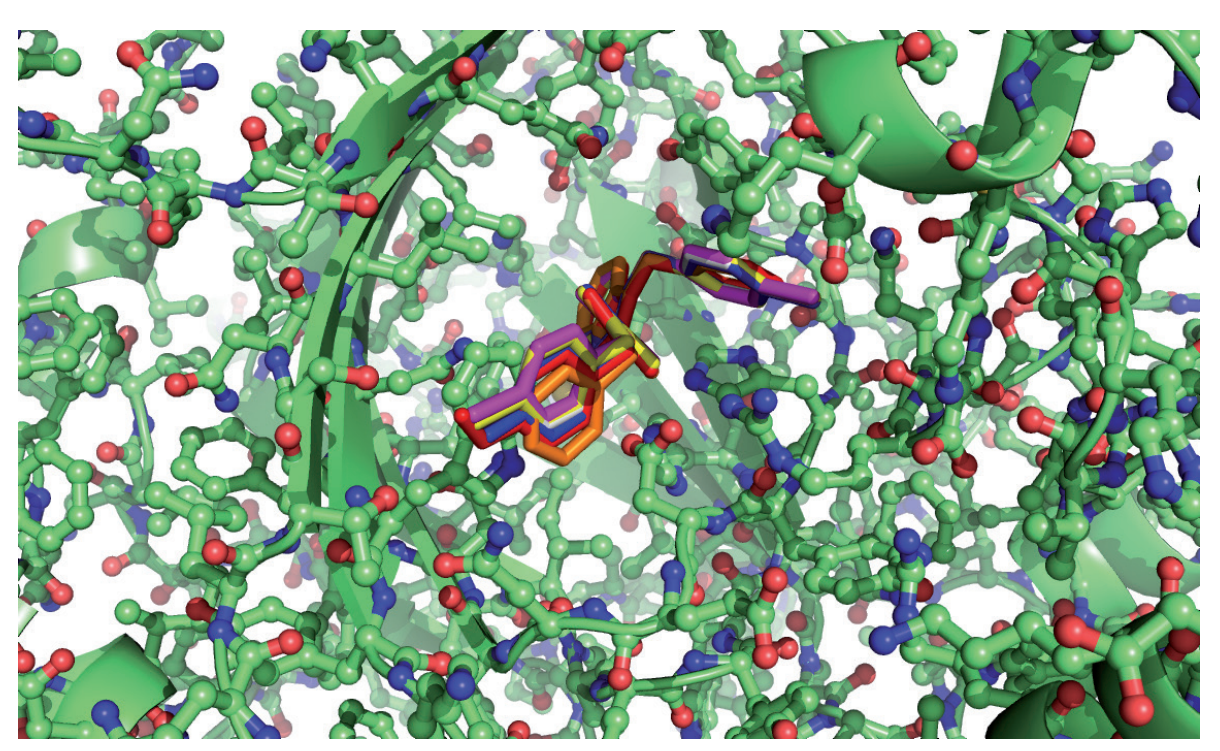

Figure 3. Binding modes of $\mathrm{H} 7$ with HIFla. Ligands are shown in stick models, while the receptor is shown in ribbon cartoon with ball and stick model. (See online version for color Figure 3).
The overall binding modes are shown in Figure 3 (including all the compounds), these compounds bind at the active site of HIF1a.

Figure 4 is the predicted binding mode of compound H7 with HIF1a. The benzimidazole ring functions as an anchor and forms hydrophobic interactions with the surrounding hydrophobic residues, including Leu186, Leu188, Phe207, and Ile281. The next important interaction is the two $\pi-\pi$ interaction formed between the compound and HIF1a. The first one is between the benzene ring of the compound and the indole ring of Trp296. The other benzene ring forms a partial $\pi-\pi$ interaction with the benzene ring of Tyr 102 . Hydrogen bonds were formed between the belly part of the compound and carboxyl group of Asp201.
The predicted binding mode of H6 is shown in Figure 5, the main interactions between the compound and the receptor do not change too much compared with compound H7.

\section{MD simulation results}

To further understand the interactions between the analogues and HIF1 $\alpha$, MD simulations were performed in this study. To explore the dynamic stability of these three protein/ inhibitor complexes and to ensure the rationality of the sampling strategy, root-mean-square displacement (RMSD) values of the protein backbone atoms during the production phase based on the starting snapshots were calculated and plotted in Figure 6. The RMSD plots indicate that the

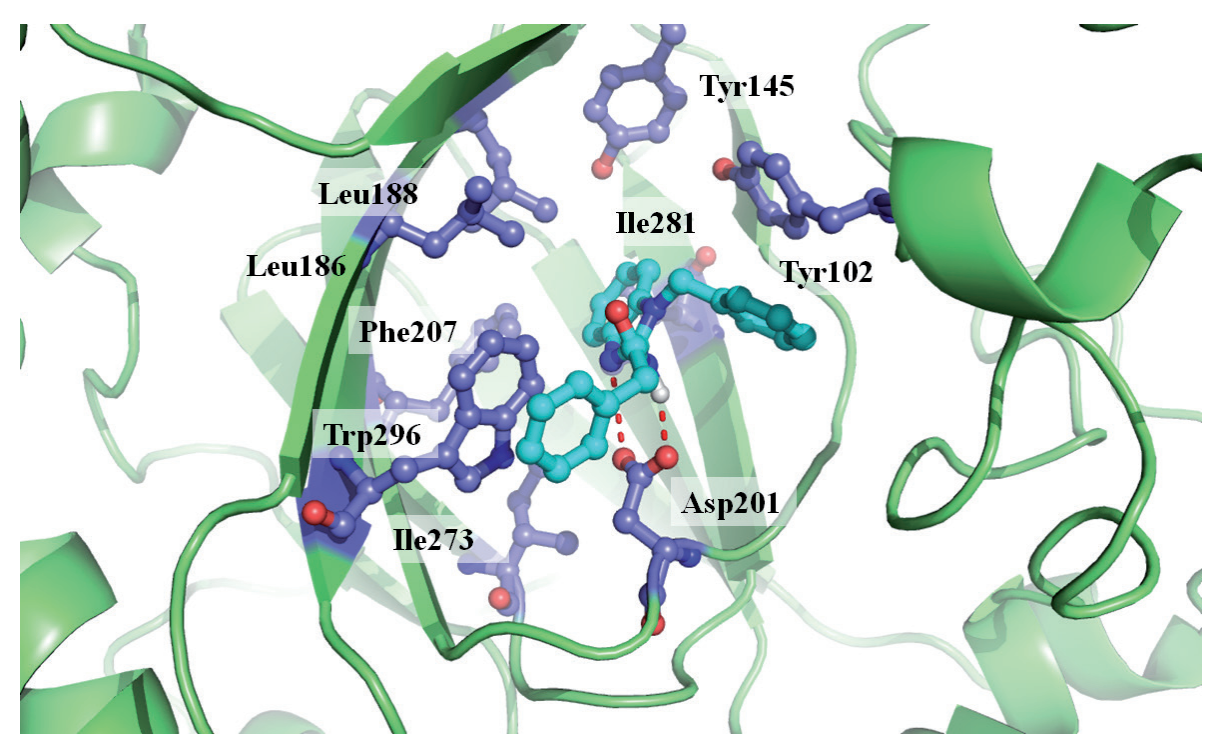

Figure 4. Binding mode of compound $\mathrm{H} 7$ with HIF1 $\alpha$. H7 is shown in cyan ball and stick model, while the protein is shown in orange ribbon cartoon. Those important residues involved in the interaction are shown in blue ball and stick model. Hydrogen bond is labeled in red dot lines. (See online version for color Figure 4). 


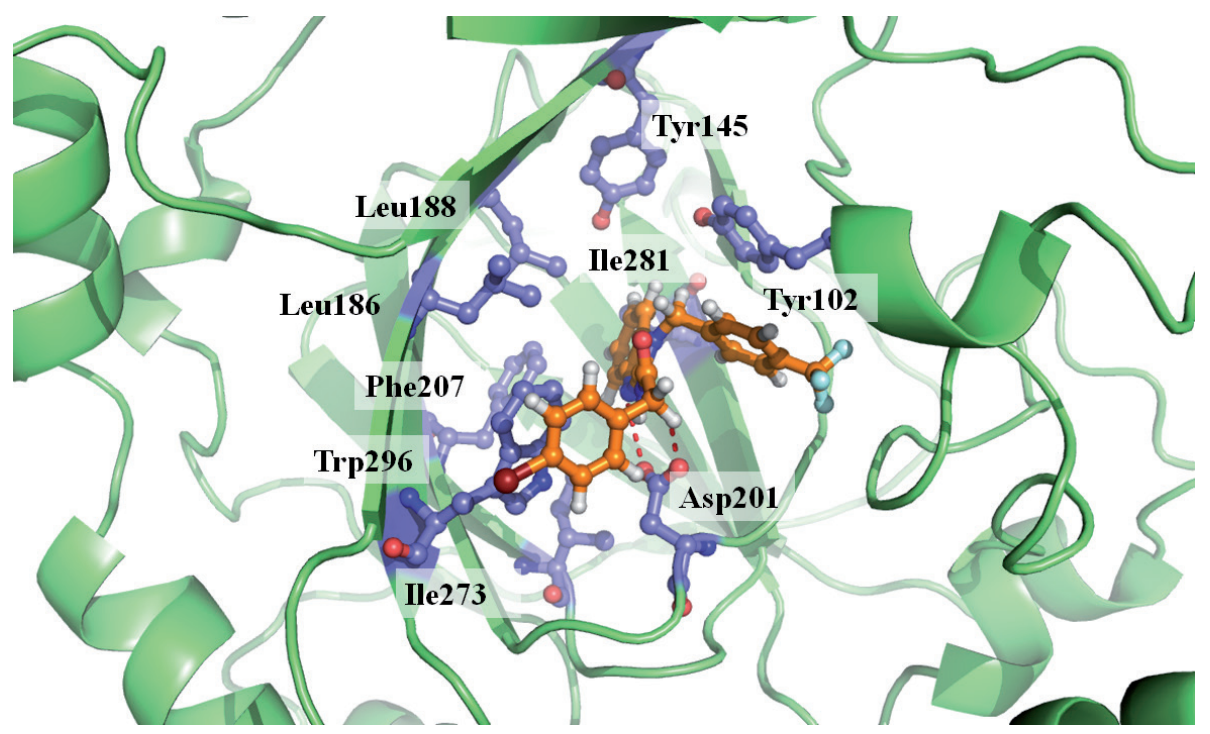

Figure 5. Binding mode of compound H6 with HIF1a. Compound H6 is shown in ball and stick model, while the protein is shown in green ribbon cartoon. Those important residues involved in the interaction are shown in stick model. Hydrogen bond is labeled in red dot lines. (See online version for color Figure 5).

Table 2. Free energy result and energy components contributing to the binding free energy $(\mathrm{kcal} / \mathrm{mol})$

\begin{tabular}{|c|c|c|c|c|c|c|}
\hline Complex & $\Delta \mathrm{E}_{\mathrm{Vdw}}$ & $\Delta \mathrm{E}_{\text {ele }}$ & $\Delta \mathrm{G}_{\mathrm{GB}}$ & $\Delta \mathrm{G}_{\mathrm{SA}}$ & $\Delta \mathrm{G}($ pred $)$ & $\mathrm{IC}_{50}(\exp )$ \\
\hline H7 & $\begin{array}{l}-23.01 \\
(0.33)\end{array}$ & $\begin{array}{l}-20.39 \\
(1.09)\end{array}$ & $\begin{array}{l}12.89 \\
(0.78)\end{array}$ & $\begin{array}{c}-3.62 \\
(0.0089)\end{array}$ & $\begin{array}{c}-34.13 \\
(0.48)\end{array}$ & 1.58 \\
\hline H6 & $\begin{array}{c}-43.88 \\
(0.39) \\
\end{array}$ & $\begin{array}{l}-22.18 \\
(1.02)\end{array}$ & $\begin{array}{l}22.41 \\
(0.70) \\
\end{array}$ & $\begin{array}{c}-6.37 \\
(0.017) \\
\end{array}$ & $\begin{array}{c}-50.04 \\
(0.43) \\
\end{array}$ & 12.45 \\
\hline
\end{tabular}

$\Delta \mathrm{E}_{\mathrm{Vdw}}$, the energy of van der Waals interaction; $\Delta \mathrm{E}_{\text {ele }}$, the energy of electrostatic interaction; $\Delta \mathrm{G}_{\mathrm{GB}}$, the polar contribution of desolvation computed using the generalized Born (GB) model; $\Delta \mathrm{G}_{\mathrm{SA}}$, the nonpolar contribution of desolvation computed using the surface area (SA); $\Delta \mathrm{G}$, the free binding energy. The predictions do not include the contribution of conformational entropy; $\mathrm{IC}_{50}$ (exp), experimental value of $\mathrm{IC}_{50}$ (half maximal inhibitory concentration).

conformations of the complexes usually achieve equilibrium at $\sim 3 \mathrm{~ns}$.

In MM/GBSA calculations, the affinity of a ligand binding to a protein can be estimated by the snapshots from a trajectory of the complex (single-trajectory protocol). The binding free energies and the energy components of the complexes are shown in Table 2.

\section{Discussion}

According to the experimental results, these seven compounds (see Figure 2 and Table 1 for detail) can be roughly sorted into two types: template compound (H7) which has the weakest binding affinity and analogues which have better binding affinity $(\mathrm{H} 1-\mathrm{H} 6)$. From the structure point of view, these analogues were designed with modifications mainly in hydrophobic substitution on two benzene rings of template compound $\mathrm{H} 7$.

According to the docking results in Table 1, Autodock Vina binding energies didn't not show significant difference here, but the template compound $\mathrm{H} 7$ does have the highest energy compared with other analogues. So from the energy point of view, these compounds can be quickly evaluated which can be very helpful in future rational drug design, saving plenty of time and resources (Stigliani et al. 2012).

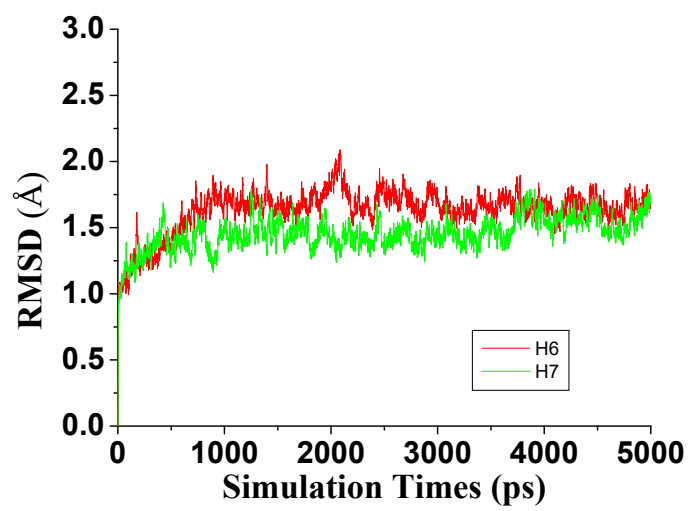

Figure 6. Root-mean-square displacement (RMSD) values of the protein backbone atoms during the production phase based on the starting snapshots. (See online version for color Figure 6). 
Figure 3 shows the overall binding modes of these compounds with the protein. We further analyzed two representative compounds from this series, including $\mathrm{H} 7$ which is the scaffold; and compounds $\mathrm{H} 6$ which was the one with the highest binding affinity.

We believe the reason that $\mathrm{H} 6$ is better than $\mathrm{H} 7$ in activity is the two substitution of $\mathrm{H} 6$ (bromide and trifluoromethyl). They help to maintain the position of the compound. As this substitution side of the compound is facing the protein surface. The extra function groups can help to push the compound back to the inside of the binding site. This can also explain why the other analogues of $\mathrm{H} 7$ all have a better binding affinity.

The molecular docking results give us a big picture of how these compounds bind with HIFla. In brief, the compounds here can be imaged as a scorpion. The benzimidazole ring in the structure, like a tail, act as an anchor and form hydrophobic interaction with a few hydrophobic residues, including Leu186, Leu188, Phe207, and Ile28 deep inside the binding cavity. The hydrogen from the amine and imidazole, like the belly, form two hydrogen bonds with residue Asp201; for those inhibitors with better binding affinity, the substitutions on the benzene ring, like two claws, help to maintain the position of the compound and form $\pi-\pi$ interaction (one is partial) with residues Trp296 and Tyr102.

In MM/GBSA calculations, the results further confirm the above docking result, which is not always the case. The binding free energy are well consistent with the experimental data, that weakest compound $\mathrm{H} 7$ has the highest binding free energy, the most potent compound H6 has the lowest energy. Besides that, it is apparently that van der Waals (VdW) interaction are very important here, as we can see in both $\mathrm{H} 6$ and $\mathrm{H} 7$, they are an important part of the overall energy.

\section{Conclusion}

HIF1 $\alpha$ as a novel anti-cancer target has been investigated by many studies. A recent published paper reported a promising new series of compound. In the present study, molecular docking calculations and molecular dynamics simulation (with MMGBSA) were used to understand the binding mechanism. According to the results, we proposed a possible binding mode for these inhibitors. And we find a few protein residues that can form key interaction with the inhibitors. Beside, through MMGBSA, we understand that the VdW interaction is critical too. This information can all be used to guide the future HIFla inhibitor design. Moreover, the method used in this study can enable us to understand that commuter aided drug design is a very useful tool in the rational drug design field.

Conflict of interest. The authors declare no conflict of interest.

\section{References}

Brown JM, Wilson WR (2004): Exploiting tumor hypoxia in cancer treatment. Nat. Rev. Cancer 4, 437-447

https://doi.org/10.1038/nrc1367

Case DA, Darden TA, Cheatham TE III, Simmerling CL, Wang J, Duke RE, Luo R, Walker RC, Zhang W, Merz KM, et al. (2006): AMBER 9, University of California, San Francisco

Chen J, Wang J, Schwab LP, Park KT, Seagroves TN, Jennings LK, Miller DD, Li W (2014): Benzimidazole analogs as potent hypoxia inducible factor inhibitors: synthesis biological evaluation and profiling drug-like properties. Anticancer Res. 34, 3891-3904

Essmann U, Perera L, Berkowitz ML, Darden T, Lee H, Pedersen LGA (1995): Smooth particle mesh Ewald method. J. Chem. Phys. 103, 8577-8592

https://doi.org/10.1063/1.470117

Forouzanfar MH, Afshin A, Alexander LT, Anderson HR, Bhutta ZA, Biryukov S, Brauer M, Burnett R, Cercy K, Charlson FJ, et al (2015): Global regional and national comparative risk assessment of 79 behavioural environmental and occupational and metabolic risks or clusters of risks in 188 countries 1990-2013: a systematic analysis for the Global Burden of Disease Study 2013. Lancet 388, 1659 https://doi.org/10.1016/S0140-6736(16)31679-8

Giaccia A, Siim BG, Johnson RS (2003): HIF-1 as a target for drug development. Nat. Rev. Drug Discov. 2, 803-811 https://doi.org/10.1038/nrd1199

Hanwell MD, Curtis DE, Lonie DC, Vandermeersch T, Zurek E, Hutchison GR (2012): Avogadro: an advanced semantic chemical editor visualization and analysis platform. J. Chem. Informatics 4, 17 https://doi.org/10.1186/1758-2946-4-17

Hsu HK, Juan SH, Ho PY, Liang YC, Lin CH, Teng CM, Lee WS (2003): YC-1 inhibits proliferation of human vascular endothelial cells through a cyclic GMP-independent pathway. Biochem. Pharmacol. 66, 263-271 https://doi.org/10.1016/S0006-2952(03)00244-2

Majmundar AJ, Wong WJ, Simon MC (2010): Hypoxia-inducible factors and the response to hypoxic stress. Mol. Cell 40, 294-309 https://doi.org/10.1016/j.molcel.2010.09.022

Masoud GN, Wang J, Chen J, Miller D, Li W (2015): Design synthesis and biological evaluation of novel H1F1a inhibitiors. Anticancer Res. 35, 3849-3859

Morris GM, Huey R, Lindstrom W, Sanner MF, Belew RK, Goodsell DS, Olson AJ (2009): AutoDock4 and AutoDockTools4: Automated docking with selective receptor flexiblity. J. Comput. Chem. 30, 2785-2791 https://doi.org/10.1002/jcc.21256

Onnis B, Rapisarda A, Melillo G (2009): Development of HIF-1 inhibitors for cancer therapy. J. Cell Mol. Med. 13, $2780-2786$ https://doi.org/10.1111/j.1582-4934.2009.00876.x

Ryckaert JP, Ciccotti G, Berendsen HJC (1977): Numerical integration of the cartesian equations of motion of a system with constraints: Molecular dynamics of $\mathrm{n}$-alkanes. J. Comput. Phys. 23, 327-341 
https://doi.org/10.1016/0021-9991(77)90098-5

Semenza GL (2000): HIF-1 and human disease: one highly involved factor. Genes Dev. 14, 1983-1991

Semenza GL (2001): HIF-1 and mechanisms of hypoxia sensing. Curr. Opin. Cell Biol. 13, 167-171 https://doi.org/10.1016/S0955-0674(00)00194-0

Semenza GL (2003): Targeting HIF-1 for cancer therapy. Nat. Rev. Cancer 3, 721-732 https://doi.org/10.1038/nrc1187

Semenza GL (2007): Hypoxia-inducible factor 1 (HIF-1) pathway. Science's STKE 407, cm8 https://doi.org/10.1126/stke. $4072007 \mathrm{~cm} 8$

Soeda A, Park M, Lee D, Mintz A, Androutsellis-Theotokis A, McKay RD, Engh J, Iwama T, Kunisada T, Kassam AB, et al. (2009): Hypoxia promotes expansion of the CD133-positive glioma stem cells through activation of HIF-1alpaha. Oncogene 28, 3949-3959 https://doi.org/10.1038/onc.2009.252

Stigliani JL, Bernardes-Genisson V, Bernadou J, Pratviel G (2012): Cross-docking study on InhA inhibitors: a combination of Autodock Vina and PM6-DH2 simulations to retrieve bio-active conformations. Org. Biomol. Chem. 10, 6341 https://doi.org/10.1039/c2ob25602a

Sullivan R, Graham CH (2007): Hypoxia-driven selection of the metastatic phenotype. Cancer Metastasis Rev. 26, 319-331 https://doi.org/10.1007/s10555-007-9062-2

Taabazuing CY, Fermann J, Garman S, Knapp MJ (2016): Substrate promotes productive gas binding in the a-ketoglutaratedependent oxygenase FIH. Biochemistry 55, 277-286 https://doi.org/10.1021/acs.biochem.5b01003
Trott O, Olson AJ (2010): AutoDock Vina: improving the speed and accuracy of docking with a new scoring function efficient optimization and multithreading. J. Comput. Chem. 31, 455-461

Wang GL, Jiang BH, Rue EA, Semenza GL (1995): Hypoxiainducible factor 1 is a basic-helix-loop-helix-PAS heterodimer regulated by cellular $\mathrm{O} 2$ tension. Proc. Natl. Acad. Sci. USA 92, 5510-5514 https://doi.org/10.1073/pnas.92.12.5510

Wang SW, Chang YL, Peng CY, Cheng FC, Pan SL, Guh JH, Chang JH (2005): YC-1 [3- (5'-hydroxymethyl-2'-furyl)-1-benzyl indazole] inhibits endothelial cell functions induced by angiogenic factors in vitro and angiogenesis in vivo models. J. Pharmacol. Exp. 314, 35-42 https://doi.org/10.1124/jpet.105.085126

William LJ, Jayaraman C, Jeffry DM, Roger WI, Michael LK (1983): Comparison of simple potential functions for simulating liquid water. J. Chem. Phys. 79, 926-935 https://doi.org/10.1063/1.445869

Yang C, Wang W, Chen L, Liang J, Lin S, Lee MY, Ma DL, Leung $\mathrm{CH}$ (2016): Discovery of a VHL and HIF1 1 interaction inhibitor with in vivo angiogenic activity via structure-based virtual screening. Chem. Commun. (Camb) 52, 12837-12840 https://doi.org/10.1039/C6CC04938A

Yeo EJ, Chun YS, Cho YS, Kim J, Lee JC, Kim MS, Park JW (2003): A potential anticancer drug targeting hypoxia-inducible factor 1. J. Natl. Cancer Inst. 95, 516-525 https://doi.org/10.1093/jnci/95.7.516

Received: March 26, 2017

Final version accepted: August 1, 2017 